\title{
Change in country-level COVID-19 case fatality rate is associated with improved testing; no apparent role of medical care or disease-specific knowledge
}

Maia P. Smith ( $\square$ Maia.phillips.smith@gmail.com )

St. George's University

\section{Research Article}

Keywords: Test Density, Medical Spending, Medical Interventions, Infection Reduction

Posted Date: May 12th, 2021

DOI: https://doi.org/10.21203/rs.3.rs-471645/v2

License: (c) (i) This work is licensed under a Creative Commons Attribution 4.0 International License.

Read Full License 


\section{Abstract}

Observed case fatality rate (CFR) of COVID-19 has decreased since the beginning of the pandemic. Reasons for this decline include improved knowledge of COVID-19 pathogenesis, leading to improved medical care of confirmed cases. However, ascertainment also plays a role: as more low-risk individuals are tested and more mild cases identified, observed CFR will decline. Previously I showed that geographylevel CFR was cross-sectionally negatively associated with test density; here I test for similar trends within geography over six months, and check plausibility of various posited causes. Although CFR varied between geographies, its association with testing did not: in 141 geographies, CFR dropped by an average of $18 \%$ for each doubling of test density. Change in CFR within a given geography was not associated either with that geography's medical spending or with whether the bulk of cases occurred early or late in the pandemic. This shows that medical interventions, including those specific to COVID-19, have only a minimal effect on total CFR. Two major conclusions follow. First, interventions to reduce CFR should be evaluated by comparing groups that received the intervention to those who did not: decline in CFR after an intervention is not evidence of effectiveness. Second, improving clinical care of confirmed COVID-19 cases has only a minimal effect on death rates. To minimize the total death toll of COVID-19, policymakers should prioritize reducing infections.

\section{Introduction}

Observed case fatality rate (CFR) of COVID-19 has dropped since the beginning of the pandemic, from $10 \%$ to $3 \%$ in the US ${ }^{1}$ and similarly elsewhere. ${ }^{2}$ Cited reasons for this decline include improved understanding of disease pathogenesis leading to better treatment, as well as the abandonment of ineffective therapies. ${ }^{2}$ However, ascertainment bias almost certainly also plays a role: early in the pandemic, tests were reserved for individuals at high risk of complications and many low-risk cases were missed. Seroprevalence data from early in the pandemic, ${ }^{3}$ when CFR in the US based on testing approached $10 \%,{ }^{1}$ suggested that when all cases were included true CFR was usually below $1 \%$ and often below $0.50 \%$; it has already dropped below $2 \%{ }^{1}$

It has already been shown ${ }^{4}$ that CFR of COVID-19 is lower in geographic regions where test density is higher. This association is similar on all continents, even though baseline CFR is higher on some continents than others. However, these cross-sectional data are confounded by geography-level factors such as population age structure, presence of comorbidities, and access to critical care. Thus while it is likely that ascertainment bias plays a role in the observed drop in CFR as test density increases, the size and consistency of this effect is not known.

The current paper investigates the role of test density in driving changes in observed geography-level CFR over a six-month period. As alternative explanations for observed changes in CFR I consider per capita medical spending as an indicator of critical-care access; and timing of the pandemic as an indicator of each geography's ability to benefit from rapidly-evolving knowledge. This technique eliminates variation 
in CFR driven by geography-level factors, and thus allows an unbiased estimate of the role of ascertainment in driving observed decreases in COVID-19 lethality.

\section{Methods}

\section{Study Design and Data Collection}

I estimate the slope of the association between change in test density, and change in observed CFR, in resolved cases of COVID-19 between July 3, 2020 and January 5, 2021. I then investigate the role of two factors (timing of the epidemic and medical spending) in driving this change.

COVID-19 data are from every geography for which they were available from a publicly-available real-time counter $^{1}$ and in which at least one death had occurred by the earlier timepoint. Timing of the epidemic was expressed as the percent of total cumulative cases at the later timepoint that occurred after the earlier timepoint. Medical spending (USD per capita, 2015) was downloaded from the World Health Organization. ${ }^{5}$

\section{Statistical Analysis}

For each geography I calculate changes in CFR (ratio between total deaths and total resolved cases) and test density per thousand residents, between the two timepoints. These data are presented as summary statistics and in corrected linear models. These models used log-log linear regression weighted by log average number of cases (mean of case count at the two timepoints.) Outputs were then reversetransformed and expressed as percent change in CFR per unit change in predictor (e.g. per doubling of test density.) I also estimate the effect of other predictors (timing of the pandemic or medical spending) on CFR change.

Change in CFR, change in test density, and total cases were all approximately log-normally distributed and thus were log-transformed for normality in all analyses. Timing of the epidemic (percent of total cases that occurred after July 3) was skewed and thus it was categorized into early, middle, and late based on $10^{\text {th }}$ and $50^{\text {th }}$ percentiles.

\section{Results}

In countries with complete COVID-19 data ( $\mathrm{n}=162)$ median CFR was $4.08 \%$ (IQR $2.2-6.9)$ on July 3 and $2.04 \%(1.3-3.1)$ on January 5. Median test density was 23 tests per thousand population (IQR $6.6-60$ ) at the earlier timepoint, and $1200(274-5000)$ at the later timepoint. A median of $88 \%$ of total cases occurred after July 3 (IQR $83-94 \%$; $10^{\text {th }}$ percentile, 48\%.)

Data on medical spending was available for 141 geographies, which included $99.8 \%$ of COVID-19 cases. Median medical spending was $\$ 1010$ per person per year (IQR $350-2200$.) 
Of those 141 geographies, CFR decreased in 120 (85\%) which contained 95\% of cases; and because test density was cumulative, it increased in every geography. For each doubling in test density, mean CFR decreased by $18 \%$ (Figure $1\left(p<0.0001, R^{2} 0.08\right.$.)

Neither medical spending nor timing of the pandemic was associated with change in CFR (Figure 2.) This null association was supported by linear regression models (both $p>0.10$ ).

\section{Discussion}

Country-level case fatality rate of COVID-19 decreased as test density increased, and on average this decline was almost the same as was previously observed between geographies at a single timepoint. ${ }^{4}$ This further supports the role of improved ascertainment in driving it. In contrast, rate of CFR decline did not vary with either access to medical care or existence of COVID-19-specific knowledge.

These results are unexpected because COVID-19 treatment undoubtedly improved during the study period. Treatments previously approved for emergency use, such as hydroxychloroquine, ${ }^{6}$ were shown to be ineffective and consequently abandoned; while therapies such as pronation and continuous positive airway pressure (CPAP) have been shown to be effective and implemented widely. ${ }^{7}$ This contrast suggests one possible explanation for the lack of observed effect of country-level medical-care access: if some treatments increase survival and some decrease it, the net effect of care access may be neutral.

This also may explain the lack of effect of timing of the pandemic. If treatment quality and consequent survivorship have improved over time, CFR should decrease more sharply in countries where most cases occurred after those improvements became public. This did not occur. In addition to low statistical power, this may be due to the relatively small number of COVID-19 cases who can benefit from improvements in care. These improvements benefit only those patients whose symptoms are severe enough to warrant COVID-specific medical care, but not so severe as to be beyond help. Since most cases are asymptomatic

8 and able to recover without COVID-specific care, this group is a minority. Thus while treatment improvements certainly benefit the patients who receive them ${ }^{7}$ they may not have a detectable effect at the population level.

\section{Conclusions}

These findings have two major implications. First, research should explicitly consider the role of ascertainment bias. Evaluation of interventions should compare groups that received it to those who did not, rather than interpreting a decline in CFR after an intervention ${ }^{9}$ as evidence of effectiveness. Second, improvements in clinical care appear to have only a minor effect on case fatality rate. To minimize the death toll of COVID-19, the priority should be to reduce infection.

\section{References}


1 Worldometers.info. COVID-19 Coronavirus Pandemic,

<https://www.worldometers.info/coronavirus/> (2020).

2 Ledford, $\mathrm{H}$. in Nature (2020).

3 Ioannidis, J. The infection fatality rate of COVID-19 inferred from seroprevalence data. Bulletin of the World Health Organization, doi:https://www.medrxiv.org/content/10.1101/2020.05.13.20101253v2 (2020).

4 Smith, M. Role of ascertainment bias in determining case fatality rate of COVID-19. J Epidemiol Global Health, doi: https://doi.org/10.2991/jegh.k.210401.001 (2021).

5 in Data Explorer (World Health Organization, 2021).

6 Axfors, C., Schmitt, A.M., Janiaud, P. et al. . Mortality outcomes with hydroxychloroquine and chloroquine in COVID-19 from an international collaborative meta-analysis of randomized trials. Nat Commun 12 (2021).

7 Ramirez, G. B., EP; Castelli, E; Marinosci, A; Angelillo, P; Damanti, S; Scotti, R; Gobbi, A; Centurioni, C; Di Scala, F; Morgillo, A; Castagna, A; Conte, C; Assanelli, A; De Cobelli, F; Calcaterra, B; Cabrini, L; Carcó, F; Turi, S; Silvani, P; Dagna, L; Zangrillo, A; Landoni, G; Tresoldi, M; Covid-19 Bio, B. Study Group. Continuous positive airway pressure and pronation outside the intensive care unit in COVID 19 ARDS. Minerva Med, doi:10.23736/s0026-4806.20.06952-9 (2020).

8 Oran, D. T., EJ. Prevalence of Asymptomatic SARS-CoV-2 Infection: A Narrative Review. Annals of Internal Medicine June 3, M20-3012 (2020).

9 Kenyon, C. Flattening-the-curve associated with reduced COVID-19 case fatality rates- an ecological analysis of 65 countries. Journal of Infection 81, e98-e99 (2020).

\section{Declarations}

Funding: none

Conflicts of interest/Competing interests: none

Availability of data and material (data transparency): publicly available at cited links

Code availability: on request

Authors contributions: MPS did everything

Ethics approval: not human-subjects research

Consent to participate: $\mathrm{n} / \mathrm{a}$ 


\section{Figures}

\section{Case fatality rate of COVID-19 declines with improved testing July 3, 2020 - January 5, 2021}

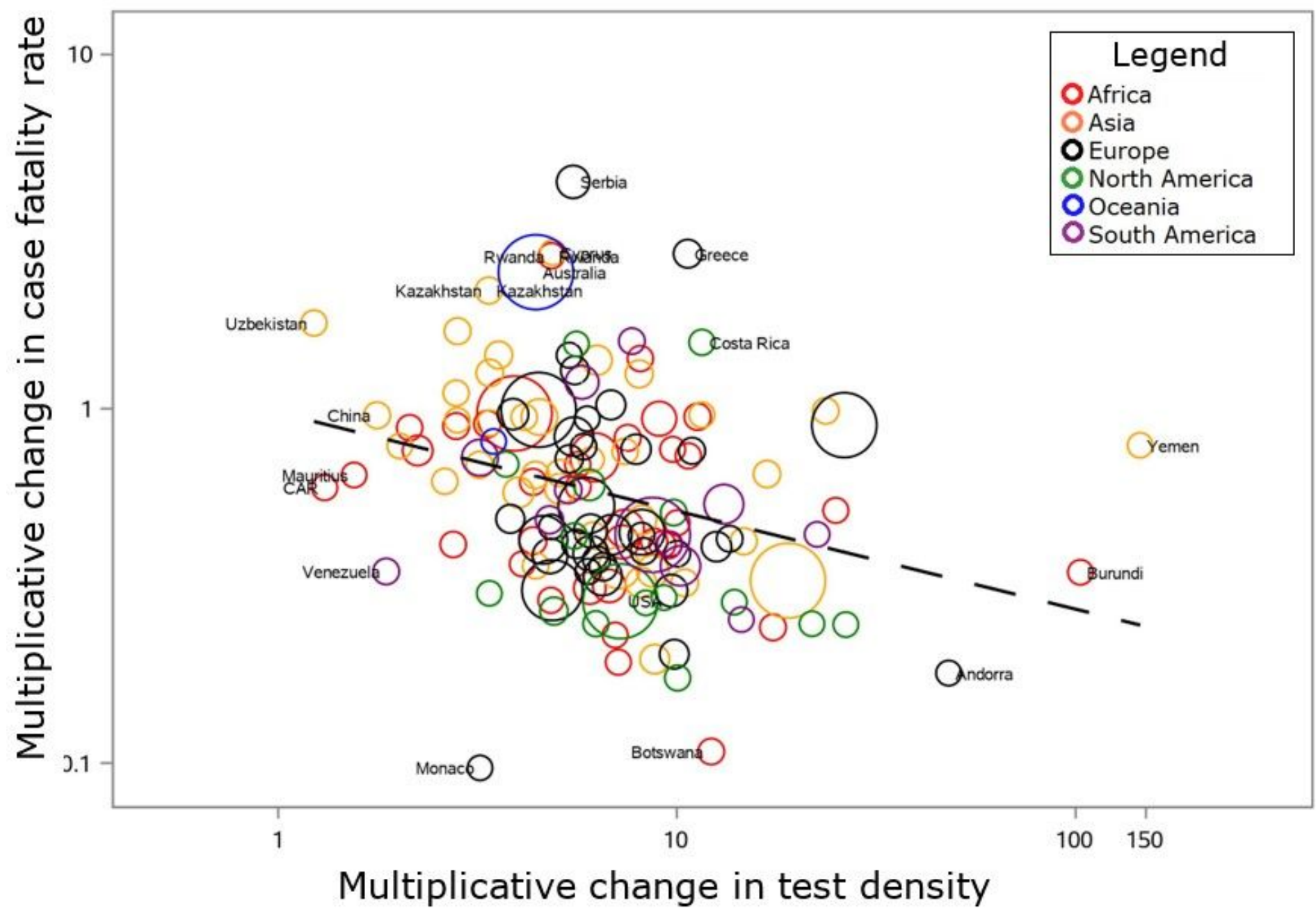

Figure 1

Case fatality rate of COVID-19 declines with improved testing Color coding for continent. Bubble size for number of cases. Regression line of best fit for $18 \%$ decrease in CFR per doubling of test density. 
Neither timing of the pandemic, nor medical care, predicts changes in COVID-19 case fatality rate

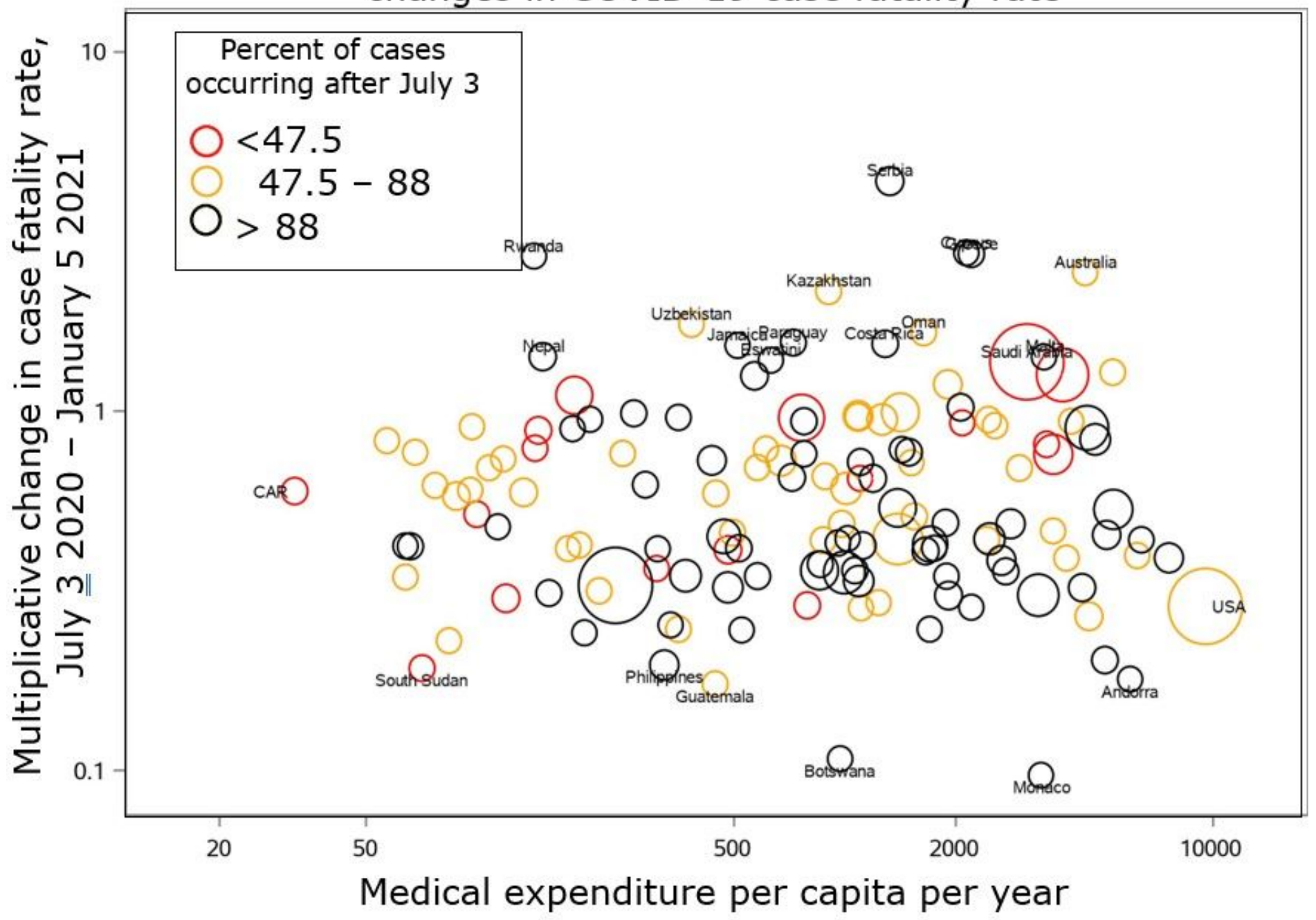

Figure 2

Changes in case fatality rate of COVID-19 are not associated with either medical spending or timing of the pandemic Color coding for timing of the pandemic. Bubble size for number of cases. 